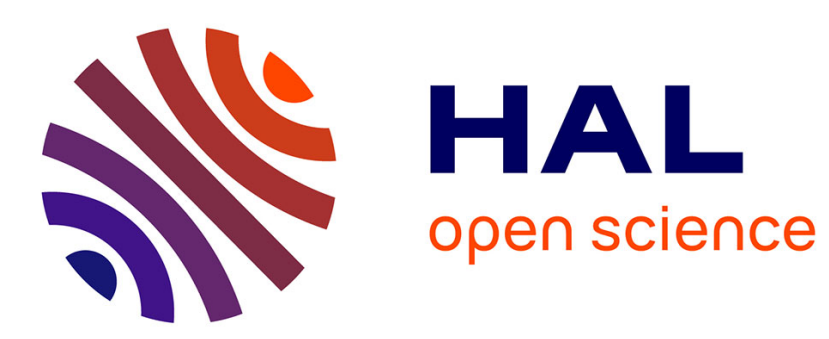

\title{
LES USINES DE LAITERIE (Fin). Quelques solutions économiques pour la conception d'industries laitières
}

\author{
P. Massaux, J. Blézat
}

\section{To cite this version:}

P. Massaux, J. Blézat. LES USINES DE LAITERIE (Fin). Quelques solutions économiques pour la conception d'industries laitières. Le Lait, 1954, 34 (337), pp.388-401. hal-00928117

\section{HAL Id: hal-00928117 \\ https://hal.science/hal-00928117}

Submitted on 1 Jan 1954

HAL is a multi-disciplinary open access archive for the deposit and dissemination of scientific research documents, whether they are published or not. The documents may come from teaching and research institutions in France or abroad, or from public or private research centers.
L'archive ouverte pluridisciplinaire HAL, est destinée au dépôt et à la diffusion de documents scientifiques de niveau recherche, publiés ou non, émanant des établissements d'enseignement et de recherche français ou étrangers, des laboratoires publics ou privés. 
P. Moglia. La vitamine antiscorbutique du lait de chèvre. Thèse doct. Ec. Méd. Vét. Alfort, 1938. Le Lait, Paris, n 178, 1938, p. 826.

E. PAGe. Les vitamines. Milan, Hoepli, 1949.

E. Parisi et G. De Vito. Ann. Chimica Appl. Vol. 24, fasc. 10, 1934, p. 504.

M. Passerini, P. Papint et E. Meloni. Quelques recherches sur les inflorescences de l'Helichrysum Italicum. Lo Sperimentale, Florence, vol. II, fasc. $3-4,1951$.

S. Pellegrini. Recherches expérimentales sur l'effet de la décoction d'Helichrysum Italicum G. Don sur le poids, sur l'éliminatoin de $\mathrm{CO}^{2}$ et sur la crase sanguine du cobaye. Ann. Fac. Méd. Vét. Un. de Pise, vol. IV, 1951.

A. Pino. Recherches sur les variations du taux du lactose dans le lait de vache pendant le travail. Le Lait, Paris, no 263-264, 1947.

L. Santini. Quelques considérations sur les effets thérapeutiques de l'Helichrysum Italicum, Castelnuovo Garfagnana, Salvietti, 1948.

L. SANTINI. Au sujet du pouvoir inhibiteur d'un extrait végétal au point de vue de quelques importantes réactions biologiques. Communication à la Société Lombarde de Sciences Médicales et Biologiques, Session du 23 décembre 1949.

E. Savini. Chimie et analyse du lait et des produits laitiers. Milan, Hoepli, 1946.

H. Selye. Le concept de Stress en 1953. Ann. Ravasini, Rome, no 4, 15 février 1954.

H. Selye. Stress (La physiologie et pathologie de l'exposition à la acontrainte systématique »). Acte d'Endocrinologie, Inc, Publ., Montréal, 1950.

E. SEREN. Recherches sur l'activité thérapeutique de l'Hélichryse en médecine vétérinaire. Inst. de Path. Spéc. et de Clin. Méd. de la Fac. de Méd. Vét. Univ. de Turin.

SpISnI. La Clinique Vétérinaire, Milan, no 12, 1945.

E. Trabucchi et ses collaborateurs : cités par Pellegrini.

\section{LES USINES DE LAITERIE (1)}

\section{par}

P. MASSAUX et J. BLÉZAT

Architectes et Ingénieurs-Conseils

(Fin)

\section{Quelques solutions économiques pour la conception d'industries laitières}

Dans un article précédent (2) consacré à la conception des bâtiments laitiers nous insistions sur un point qui nous sembledevoir attirer tout particulièrement l'attention de ceux qui étudient une création ou une transformation d'industrie laitière et disions :

(1) Le Génie rural, 1953, 1.2, 353.

(2) Le Lait, 1954, 335-336. 
“La rentabilité d'une installation laitière est fonction, évidemment, de l'importance totale de l'usine, elle-même en dépendance étroite des possibilités de chaque chaîne de fabrication, dans une région donnée, et suivant les ressources en matière première et les facilités de collecte de celle-ci.

«Il appartient donc à ceux qui étudient une création ou une transformation d'industrie laitière, de fournir tous les éléments nécessaires à l'analyse de cette rentabilité. Le problème, actuellement à l'étude sur le plan général, est aussi très complexe, surtout en notre pays ou la polyvalence est la règle presque générale.

"Une courbe de rentabilité est ascendante en fonction de la quantité, mais présente aussi de nombreux paliers et seuils en deçà desquels il faut prendre soin de se tenir.

"La souplesse d'une installation peut encore être ici un facteur important d'une bonne conception. ")

Il s'agit là d'une évidence.

Cependant, l'étude d'une courbe de rentabilité est forcément, dans notre pays où l'importance des laiteries est si variable et où la polyvalence est la règle, impossible sur le plan général.

Si bien qu'en chaque cas on en vient à une étude précise du problème soumis.

En contribution à ce genre d'étude, toujours nécessaire, nous développons un exemple relatif à une usine de laiterie desservant une ville moyenne (lait nature et fabrications annexes).

Nous avons supposé qu'une installation existait et qu'il fallait la transformer, la rénover et l'agrandir.

C'est, en effet, le cas le plus fréquent qui se présente dans un pays où le plus grand nombre d'installations existe déjà.

Nous avons donc établi (annexe n $n^{0} 2$ ) d'après quelques expériences très contrôlées, une étude économique concernant un projet moyen basé sur les prix pratiqués en 1952.

Nous avons employé pour ce projet des données réelles et recueillies dans des comptabilités déjà éprouvées, pour des usines de notre connaissance. En annexe no 1 calcul des consommations électriques prévisibles (les autres consommations se trouvent dans le corps de l'annexe no 2 ).

Cette étude nous permet de constater :

- Que les frais d'amortissement par litre de lait, pour des usines de ce genre et de cette importance, s'élevaient à environ o fr. 75 .

- Que les frais d'exploitation étaient alors de $8 \mathrm{fr} .90$ par litre, comprenant 4 fr. 10 pour les frais de ramassage (y compris amortissement du matériel roulant) et $2 \mathrm{fr}$. 50 pour frais de distribution (dito), c'est-à-dire se décomposant comme suit : 


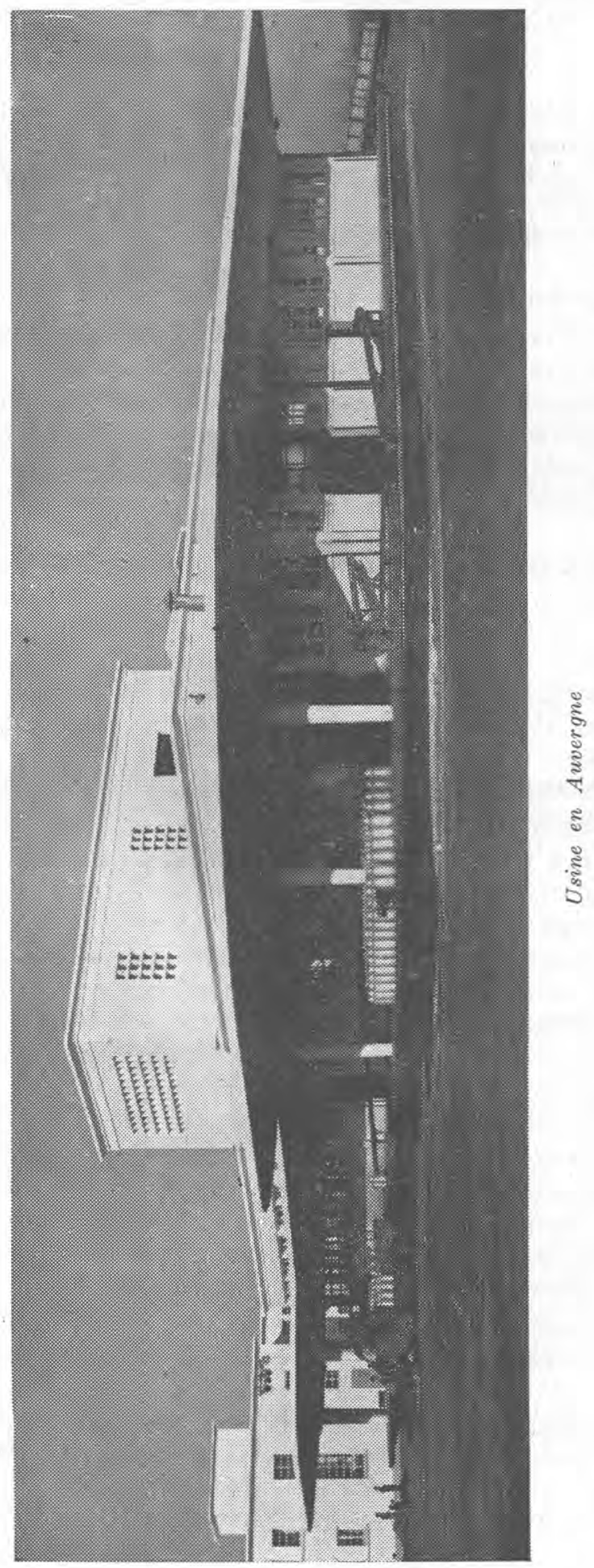


- Frais de ramassage .............

- Frais d'exploitation divers ........

- Frais de distribution ............

Munis de ces chiffres, essayons de définir les normes de rentabilité.

Toute mesure rentable doit évidemment tendre :

- A diminuer les frais d'amortissement par litre sans apporter une majoration supérieure des frais d'exploitation.

- Inversement, à diminuer les frais d'exploitation par litre sans apporter une majoration supérieure des frais d'amortissement.

Ce sont là des vérités premières, et cependant combien d'exemples prouvent qu'on s'éloigne souvent de leur application.

\section{Diminution des frais d'amortissement par litre}

Là encore, il est évident qu'on ne peut diminuer ces frais que de deux façons :

- Augmenter le litrage pour le porter au maximum (exploitable dáns l'installation);

- Limiter au minimum, sans nuire à la qualité, les frais de premier établissement (bâtiments et équipements) pour le traitement de la quantité fixée.

\section{Augmentation du litrage}

C'est là une mesure qui est du ressort de l'administration de l'usine, mais il n'est cependant pas interdit, à ceux qui sont appelés à conseiller cette administration, d'attirer son attention sur la prudence avec laquelle cette augmentation doit être recherchée, et notamment sur les inconvénients éventuels suivants, dont la nomenclature peut se déduire de l'examen des articles constituant la liste des frais d'exploitation :

- Souvent le désir de l'augmentation du litrage conduit inconsidérément à ramasser du lait dans des zones éloignées, ou bien dans lesquelles la densité kilométrique de ramassage est faible, d'où, en l'un et l'autre cas, une forte majoration des frais de ramassage, chapitre capital de ces frais d'exploitations.

- Souvent ce même désir ne tient pas assez compte des possibilités maxima pendant la période optima (été en général) et conduit alors, comme nous l'avons vu souvent, à des mesures d'exploitation extraordinaires pendant cette période, mesures qui s'avèrent d'une rentabilité fort douteuse, d'autant plus qu'au cours de cette même période les conditions économiques sont peu favorables à des écoulements avantageux des produits. 
D'où il résulte qu'on est alors quelquefois entraîné à de nouvelles majorations d'installations, majorant à leur tour les frais d'amortissement, et faisant souvent perdre ainsi le bénéfice dû à l'augmentation de litrage.

Nous l'avons vu très souvent, récemment.

C'est un cercle vicieux dans lequel se sont trouvées entraînées des organisations qui risqueraient fort de le regretter au retour de cycles économiques moins favorables, par exemple deux ou trois années de sous-consommation de produits laitiers (excédent de fruits rouges, pouvoirs d'achat affaiblis, etc.).

On en arrive ainsi à une majoration de tous les articles de notre étude économique. Les amortissements sont majorés, les frais de ramassage, les consommations également, etc.

On ne peut conclure que par des avis de prudence, quant à l'augmentation possible du litrage.

A moins, bien entendu, d'une absolue certitude de majoration de la distribution des produits fabricables.

\section{Limitation des frais de premier établissement}

Elle ne peut guère porter, évidemment, que :

- Sur les constructions ;

- Sur l'équipement.

Sur les constructions, elle ne peut porter que sur la conception d'une part, et sur les matériaux mis en œuvre, d'autre part.

Pour développer ces considérations, il faudrait une place considérable. Cependant, nous reportons à la note d'étude que nous avons précédemment établie, et résumant quelques idées sur les dispositions générales en fonction des nécessités de chaque problème.

Mais, là aussi, nous préciserons l'importance d'établir préalablement un programme judicieux, car les nécessités auxquelles nous faisons allusion ci-dessus sont souvent définies avec l'un des deux défauts classiques :

- L'insuffisance (en période critique), qui conduit malgré toutes les précautions prises pour des majorations possibles, à des installations devenant rapidement caduques et dont les amortissements sont alors inappropriés ;

- L'excès (en période d'euphorie) qui conduit naturellement à un excès proportionnel de ces amortissements.

En ce qui concerne les matériaux utilisables nous mettrons l'accent sur la nécessité d'un choix très poussé. Nous y avons fait allusion dans la note précédente, où nous avons traité des sols, murs, etc. 
Nous disions aussi que des essais étaient en cours. Nous parlions de quelques-uns (matériaux de remplacement pour murs, peintures). Actuellement ces essais ont eu quelques résultats. Nous reviendrons ultérieurement sur ceux des briques murales à parements lavables, sans apposition de revêtement. Nous joignons (annexe $n^{\circ} 3$ ) quelques résultats d'essais sur certaines peintures susceptibles de remplacer économiquement les revêtements, tout en rendant plus faciles les adaptations éventuelles de locaux, par suite de la même économie des transformations possibles.

D'autres peintures, améliorant celles essayées, et permettant en particulier un entretien plus facile sur murs humides, nous sont présentées et vont être à leur tour essayées.

Nous ne nous étendrons pas davantage sur les réductions possibles des frais de premier établissement des bâtiments, car il est évident que, outre les généralités ci-avant, tout n'est, dans leur étude, que soin dans leur bonne adaptation au programme, et solutions de constructions présentant l'économie maximum, compte tenu de cette adaptation.

Sur l'équipement, la limitation possible des frais de premier établissement est fonction : fixé ;

- Du choix de cet équipement, compte tenu du programme

- De ce choix, pièce par pièce, en tenant compte de l'usage demandé ;

- De ce choix, pièce par pièce, pour tendre au maximum à la réduction des frais d'exploitation entraînés, suivant leur débit et leurs consommations.

Dans cette note générale, nous ne passerons pas en revue la totalité du matériel utilisé en laiterie, ce qui nécessiterait un volume.

Nous donnerons done seulement quelques points de vue personnels :

\section{A) EN FONCTION DU PROGRAMme FIXÉ :}

Se méfier de la tendance à vouloir faire "une belle installation " par l'emploi d'un équipement superflu.

Depuis le choix de l'équipement du quai de réception jusqu'à celui des ehambres de stockage des produits finis, on recueille souvent des demandes abusives, surtout en période économique favorable.

Depuis la bascule de deux tonnes dans laquelle on pèse le lait de quelques bidons seulement, en passant par la laveuse de pots pouvant faire tout le travail en une demi-heure, par l'écrémeuse au débit superflu, le matériel de beurrerie utilisé au tiers de sa puis- 
sance, comme celui de la fromagerie, et en arrivant à des frigorifiques trop importants, les exemples abondent de cette inadaptation du matériel au programme.

Nous connaissons une laiterie qui ne pourra jamais débiter plus de 15.000 bouteilles de lait par jour, mais se trouve munie d'une superbe installation de 6.000 bouteilles à l'heure, que les locaux permettraient de doubler facilement.

Alors que, par ces économies possibles reportées sur quelques moyens de manutentions, ou le remplacement d'un générateur, l'acquisition de quelques condensateurs, on pourrait non seulement arriver à des économies de main-d'œuvre et de consommation, mais aussi, dès l'abord, diminuer au total fortement les frais de premier établissement et par conséquent leur amortissement.

B) EN TENANT COMPte de L'USAge DEMANDÉ, SE MÉfier, LA ENCORE, DE L'INUTILE.

On critique souvent la rusticité de certaines fabrications de matériel, en prétendant que la solidité est victime de cette conception.

Il nous prend envie, à cette occasion, de reporter à nos considérations concernant les parties "dures " des bâtiments et celles pour lesquelles peuvent être utilisés des matériaux légers suivant qu'on se trouve en présence de parties dont la transformation doit ou non être prévue étant donné le caractère très évolutif de l'industrie.

Il en est certainement de même pour l'équipement.

Le choix est alors, évidemment, bien délicat, mais on peut cependant éviter de lourdes dépenses par un emploi plus judicieux dans la simplicité comme pour certaines chaudronneries (aluminium au lieu et place d'inoxydable) ou tuyauteries (laiton étamé) ou autres installations (électricité, manutention...) ou pour des appareillages moins généraux comme les laveuses diverses (bidons, bouteilles) des barattes, etc.

Sans pour cela s'écarter de l'emploi des autres chaudronneries ou tuyauteries (inoxydables), des installations électriques traitées avec des précautions spéciales, etc., mais seulement lorsque l'usage requis les nécessite absolument (ce qui n'est pas le cas de tous ces usages) et quand des débits à traiter font présager une fatigue rapide du matériel (barattes, laveuses, etc.).

Sinon on arrivera à des installations trop coûteuses pour l'usine étudiée, ou mal amorties quand arrivera l'époque de remplacement nécessaire (accroissement ou évolution des fabrications, etc.).

Ce doit donc être là l'objet d'une étude approfondie de l'installation. 
Quand la simplicité, voire la rusticité, s'avèrent suffisantes, on peut avec avantage les adopter.

C) Pour tendre a la RÉdUCtion des fRais D'exploitation. NoUS REJOIGNONS LA LA SECONDE PARTIE DE L'EXPOSÉ, QUE NOUS ALLONS DONC ENTREPRENDRE.

\section{Diminution des frais d'exploitation par litre}

c) Nous reprenons là l'étude économique jointe (annexe $n^{0} 1$ ) dont nous parcourons les chapitres :

A) Frais de ramassage.

C'est là le plus important de nos chiffres (4 fr. 10 par litre) et chaque directeur d'usine nous a toujours semblé y attacher, très justement, la plus grande attention. Ce chiffre, dans le cas moyen envisagé, se rapporte à une densité kilométrique d'environ 12 à 14 litres par kilomètre, et comprend tous les frais relatifs à ce ramassage, dont l'amortissement du matériel roulänt, celui des récipients, les salaires, les consommations. Nous n'avons ni la compétence, ni assez d'expérience pour nous permettre un développement des causes et moyens de réduction de ce chiffre, mais pensons qu'il est réductible, eu égard à certaines autres installations dans lesquelles nous avons constaté :

- La présence de centres de collecte concentrant les apports et réduisant les horaires et kilomètrages des camions ;

- La bonne adaptation de camions par leur aménagement, leur capacité, en fonction de la " tournée " qui leur est assignée ;

- Le contrôle horaire et kilomètrique sur des camions ;

- L'entretien des véhicules effectué au maximum par les services de la laiterie.

Mais nous arrêterons là ces considérations dont nous n'avons pas les éléments pour en faire analyse.

B) Frais de consommation.

Nous écrivions ci-dessus que l'équipement devait être choisi pour tendre à la réduction des frais d'exploitation. Parmi ees derniers, les frais de consommation représentent, dans l'exemple cité, une part encore importante.

Or, il est évident que les anomalies déjà signalées conduisent à des surconsommations. L'installation d'une laveuse de 6.000 bouteilles à l'heure fonctionnant une heure à deux par jour est d'un "rendement" bien déraisonnable.

Mais ce n'est pas encore dans cette inadaptation du matériel laitier qu'on trouve les anomalies les plus grandes. C'est bien davan- 
tage dans les équipements producteurs ou distributeurs (chaleur, froid, électricité, etc.). qu'on découvre les rendements les moins étudiés.

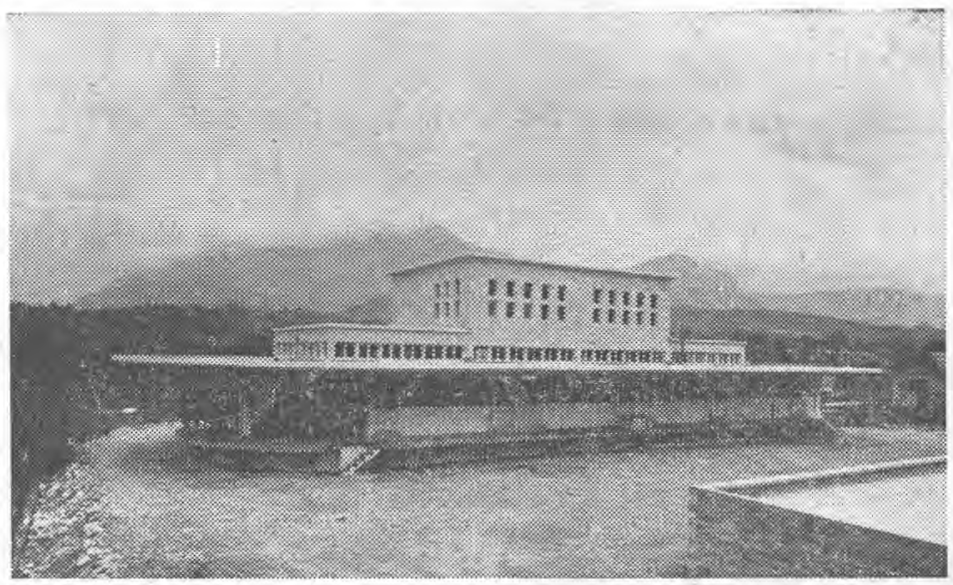

Usine en Savoie

Il n'est jamais arrivé qu'une rationalisation de chaufferie, par exemple, n'amène une économie de l'ordre de 25 à $55 \%$ pour ne citer que les exemples de notre connaissance récente.

Il n'est pas rare, en effet, que des mesures sur des installations

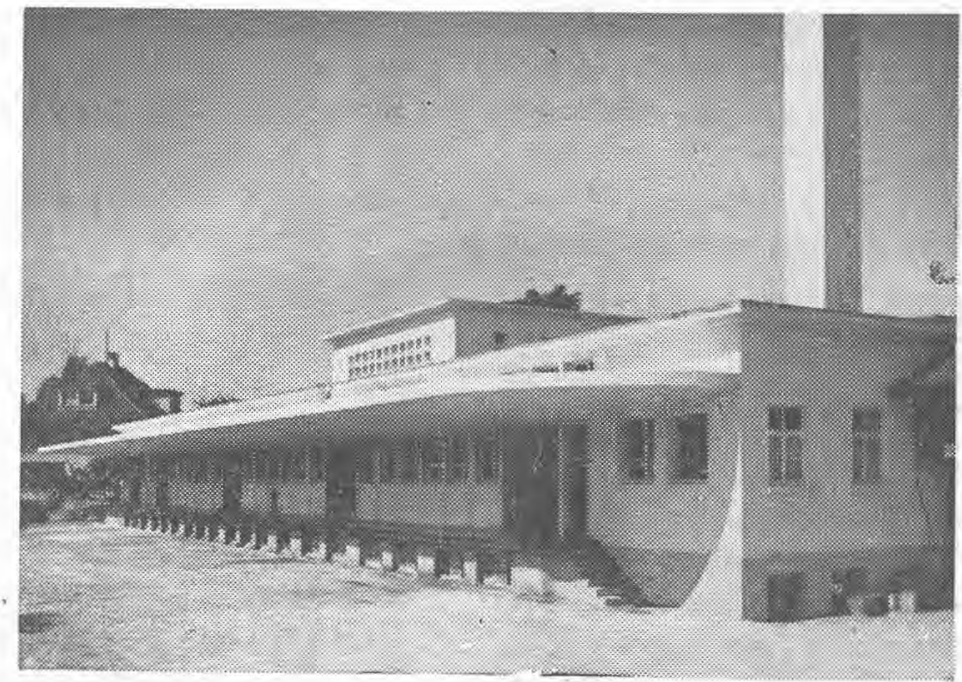

Usine dans le Haut-Rhin 
existantes aient fait ressortir des rendements de 60 et même $40 \%$ pour ces chaufferies, alors qu'une addition ou un remplacement ont porté ces chiffres à $72,76,80$ et même $84 \%$. Quatre réalisations datant de 1952 nous permettent en effet de eiter :

- Laiterie de 8 à 10.000 litres par jour ; rendement chaudière 75, 02.

- Laiterie d'environ 15.000 litres par jour ; rendement chaudière 76,12 .

- Laiterie d'environ 60.000 litres par jour; rendement chaudière 84,28 .

- Laiterie d'environ 25.000 litres par jour ; rendement chaudière 72,56 .

Ces rendements étant calculés en fonction du pouvoir calorifique supérieur du combustible utilisé et en marche normale (et non "poussée ").

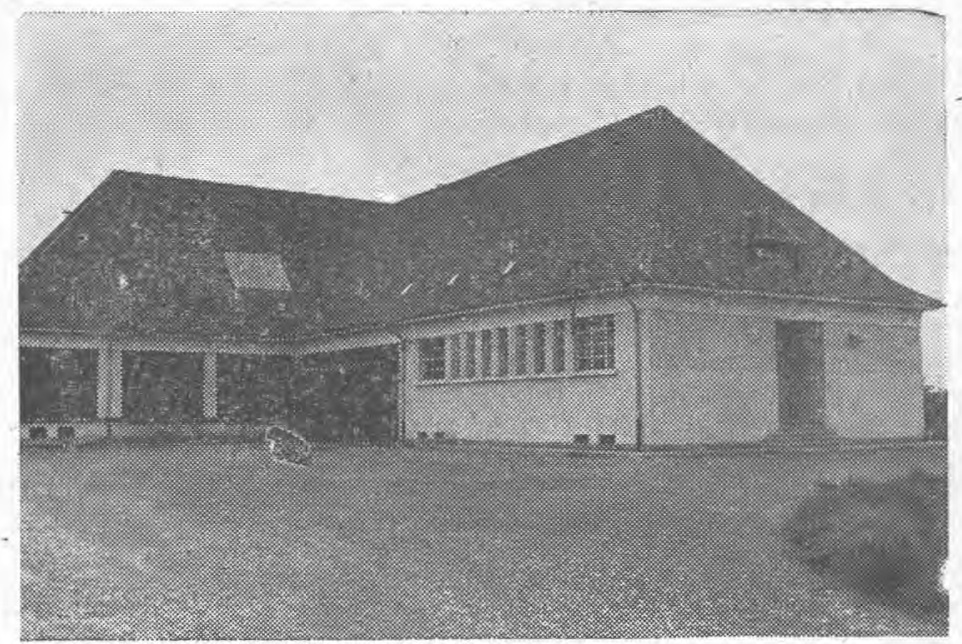

Usine en Alsace

Qu'il suffise alors de citer que la première de ces laiteries a vu ses frais de consommation baisser, sur la moyenne des mois de juin, juillet, août, septembre et octobre 1952, par rapport à la moyenne des mêmes mois de 1951, de 1.700 francs par jour.

On voit aussi combien le choix correct d'une chaufferie "adaptée " peut conduire à cette "limitation des frais d'exploitation" qui nous préoccupe. 
Mais l'autre consommation la plus importante (de l'ordre de la moitié aux trois quarts de celle de la vapeur) est l'électricité.

Et là aussi nous avons souvent pu constater combien le «souci du cos 0 " et celui de 1 'énergie réactive n'étaient pas parmi les plus grands d'une exploitation.

Une étude poussée de l'installation électrique et sa surveillance ont souvent conduit, par l'adaptation de condensateurs, la correction des lignes de distribution, la mise en état des moteurs (ces martyrs de la laiterie) à des économies de l'ordre de 20 à $35 \%$ sur la consommation (nous nous référons à des cas concrets).

Quant aux autres consommations (eau, produits d'entretien) leur économie est beaucoup moins du ressort de ceux qui conçoivent les usines et leurs installations, que de ceux qui les utilisent, et les entretiennent, pour essayer d'éviter les gaspillages. L'incidence de ces chapitres est assez minime.

Cependant nous insistons sur la question de la qualité chimique de l'eau, dont le jugement incombe bien au technicien installateur, par suite des dépôts possibles, risques de corrosions et de baisses de rendement. Là aussi une épuration, physique ou chimique, peut conduire à une sensible réduction des frais d'exploitation des appareillages utilisant l'eau incriminée.

C) Frais de main-d'auvre.

Il s'agit là d'un chapitre extrêmement important de l'étude économique jointe, puisqu'il représente $1 \mathrm{fr}$. 80 sur un total de 2 fr. 30 des frais d'exploitation (hors les ramassages et livraisons).

C'est dire combien doit être poussée l'étude d'une laiterie pour essayer de réduire ce chiffre de la façon la plus effective.

Il n'y a pas lieu, dans une installation, où l'on ne puisse influencer sur ce chiffre, par :

- Une amélioration des facilités de travail de la main-d'œuvre occupée ;

- Une facilité accrue de la surveillance du travail ;

- Une adaptation du matériel pour la diminution du nombre d'employés à son utilisation ;

- Une adaptation des débits du matériel pour l'emploi à plein d'une équipe minimum.

a) Améliorations des facilités de travail:

Ces améliorations porteront surtout sur l'étude rationnelle des circuits, la suppression des longs parcours, la mise à la vue 
directe et à leur portée des appareils de contrôle, commande, et. de travail des usagers.

Ces conditions sont d'ailleurs compatibles avec cet autre élément indispensable des laiteries qui est, au maximum, la facilité d'entretien présentée par la réduction des circuits et leur parfaite accessibilité.

\section{b) Facilité de surveillance:}

Nous en avons parlé dans la conception des bâtiments.

La proximité des services de contrôle (chefs de quais, laboratoires, maîtrise des fabrications, etc.) des lieux de travail leur incombant peut éviter des fausses manœuvres, des négligences, etc. La répartition de ces lieux de contrôle dans l'installation a done son importance.

Mais citons aussi les automatismes dans le contrôle des machines (enregistreurs thermiques, alimentation automatique de chaufferie, thermostats et sécurités diverses des frigorifiques, compteurs de lait, etc.).

\section{c) Adaptation du matériel pour diminution de la main-d'ceuvre:}

C'est là une fonction importante des techniciens procédant à la conception des programmes de matériel et au choix de celui-ci.

Ce problème se résout d'ailleurs toujours presque mathématiquement par une comparaison de la somme des frais d'amortissement et d'exploitation (main-d'œuvre et consommation) d'un équipement, aux économies de main-d'œuvre qu'il peut permettre.

La connaissance des moyens de manutention possibles, celle du temps nécessaire à un travail pour un nombre déterminé d'employés, sont les éléments nécessaires à ce jugement.

Il se peut qu'on soit alors amené à refuser tel équipement de quai dont les frais d'amortissement et de consommation excèdent l'économie de main-d'œuvre envisagée, ou au contraire à le préconiser.

C'est ainsi qu'en 1949, 1950, 1951 et 1952, ce souci nous a guidé et fait concevoir quelques-unes de ces installations génératrices de plusieurs centaines de milliers de francs d'économies annuelles pour une dépense n'excédant pas trois fois le chiffre des sommes économisées annuellement (en particulier pour les quais, les manutentions, etc.).

Mais il nous est arrivé aussi de les déconseiller quelquefois, en certains endroits, où ces équipements, très spectaculaires, 
étaient jugés désirables bien que non rationnellement adaptables.

Dans le même ordre il faut se méfier de certains matériels d'origines étrangères fort bien adaptés aux modes d'exploitation de leurs pays d'origine et ne conduisant plus à une rentabilité quand on veut les faire répondre aux sujétions de nos entreprises (formes et couvrages différents des récipients, triage et utilisation des laits acides à l'arrivée, départs de produits dans une partie des récipients, etc.

Cependant que, au contraire, leur emploi est fort indiqué en d'autres cas, avec quelques menus aménagements.

Dans le même ordre encore, l'économie de main-d'cuvre peut être sensible par l'adaptation d'un matériel amélioré, en applica. tion de techniques nouvelles ou en restant dans les usages établis.

Nous ne ferons qu'une allusion démonstrative à ce sujet aux extrêmes de nouvelles techniques de beurrerie (procédés continus) ou de fromagerie (fabrication de pâtes cuites en grandes cuves à partir de laits pasteurisés, ou machines Steinecker et similaires) mais en précisant cependant que c'est surtout dans une très bonne organisation des fabrications, même dans leur forme très traditionnelle (surtout dans la fromagerie) que peut résider l'économie maximum de main-d'œuvre. Une bonne étude des positions relatives des appareillages et surtout de la disposition des salles (égouttage, pressée, etc.), ainsi que des locaux d'affinage et de stockage, est très importante.

En Normandie, nous avons constaté, dans la fabrication éminemment traditionnelle du camembert, dans des usines d'importance sensiblement égale, une différence de 25 à $30 \%$ sur le chiffre de la main-d'œuvre occupée pour 1.000 litres de lait traité.

Il y a là certainement, une partie importante du rôle du technicien chargé de la conception.

\section{d) Adaptation des débits pour l'emploi à plein d'une main-d'ouvre minimum:}

Là encore, le choix de l'appareillage a une bien grosse importance.

La "journée» de main-d'œuvre doit, en effet, être occupée pour un rendement optimum. Précisément le lait devant en général être travaillé dans les premières heures de eette journée, il importe de choisir, certes, des débits d'appareillage en conséquence, mais aussi sans exagération. L'organisation des heures d'arrivée du lait peut permettre une meilleure répartition, mais ce n'est pas là qu'on trouvera une économie sensible de la main-d'œuvre. Tout au plus, 
une certaine sécurité supplémentaire, à chiffre égal du nombre d'employés.

Par contre sur la suite des opérations, qui peuvent être entreprises après les traitements de préparation du lait, de fortes économies sont possibles.

Le choix d'une chaîne d'embouteillage peut, par exemple, être guidé dans certaines usines de petites dimensions, par la possibilité d'utiliser, pour cette opération, l'équipe occupée, dans la matinée, au quai de la laiterie et à la pasteurisation, ceci moyennant un débit légèrement accru de la chaîne d'embouteillage, dont l'amortissement de la différence de prix est ainsi plus que largement compensé.

De même par exemple, le stockage de lait préalablement refroidi, ou pasteurisé, pour étendre ensuite sur une journée (au lieu de 2 ou 3 heures) une ligne de fabrication est souvent une rationalisation très rentable.

Le matériel doit évidemment être prévu en conséquence.

Autre exemple : une double cuisson (ou triple) en fromagerie de pâtes demi-cuites ou cuites, conduit aussi à cette utilisation maximum de la main-d'œuvre et peut-être conseillée avec un matériel conçu pour ce travail.

En beaucoup d'autres occasions, un approfondissement du problème doit permettre cette limitation au minimum des dépenses dans ce chapitre, le plus important des frais d'exploitation.

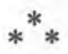

Nous avons ainsi passé en revue chacun des articles de la liste des frais d'amortissement et d'exploitation d'une installation laitière, en les rattachant, d'une façon forcément rapide et générale, à la fonction de ceux qui conçoivent les usines.

Dans chaque cas particulier, un bien grand nombre d'autres sujétions surgissent, mais nous croyons qu'en appliquant à chacune d'entre elles un raisonnement de recherche de la rentabilité pour chaque service de l'usine on doit arriver à cette économie maximum par une installation adaptée sans insuffisance ni superflu, parce que rationnelle. 
AnNexe No 1

\section{ÉTAT DES MOTEURS ET CONSOMMATIONS}

\begin{tabular}{|c|c|c|c|}
\hline Local et moteurs & Force & Durée & CV/Jour \\
\hline Chaufferte & 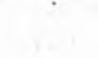 & & \\
\hline Brûleur existant $\ldots \ldots \ldots \ldots \ldots \ldots$ & 1,5 & Secours & Secours \\
\hline Pompe alimentaire existante $\ldots . .$. & 1,5 & » & " \\
\hline Pompe mazout $\ldots \ldots \ldots \ldots \ldots \ldots$ & 1,5 & 》 & 》 \\
\hline Ventilation nouvelle chaudière ..... & 1,5 & 5 & 7,5 \\
\hline Nouveau brúleur..$\ldots \ldots \ldots \ldots \ldots$ & 1 & 5 & 5 \\
\hline Pompe alimentaire $\ldots \ldots \ldots \ldots \ldots$ & 4 & 5 & 20 \\
\hline Pompe E.F. sur citerne $\ldots \ldots \ldots \ldots$ & 3 & 10 & 30 \\
\hline MachINeS A FROID & & & \\
\hline Compresseur bac à eau glacée ....... & 10 & 8 & 80 \\
\hline Compresseur bac à eau glacée....... & 10 & 8 & 80 \\
\hline Compresseur bac à saurnure ....... & 10 & 4 & 40 \\
\hline Compresseur congélation ......... & 4 & 6 & 24 \\
\hline Agitateur bac à eau glacée $\ldots . . \ldots$. & 2 & 8 & 16 \\
\hline Agitateur bac à saumure $\ldots \ldots \ldots$ & 1,5 & 4 & 6 \\
\hline Pompe à eau glacée ............ & 5 & 8 & 40 \\
\hline Pompe à saumure $\ldots \ldots \ldots \ldots \ldots$ & 1,5 & Secours & Secours \\
\hline Pompe à circulation condenseur ..... & 5 & 12 & 60 \\
\hline $\begin{array}{c}\text { Dans ce chapitre les temps de fonction- } \\
\text { nement sont les temps moyens compte } \\
\text { tenu des variations saisonnières. } \\
\text { CHAMBRES FROIDEs }\end{array}$ & & & \\
\hline Ventilateur congélation $\ldots \ldots \ldots \ldots$. & 5 & 6 & 30 \\
\hline Ventilateur chambre à bouteilles .... & 4 & 8 & 32 \\
\hline SALLE DES TANKS & & & \\
\hline $\begin{array}{c}\text { Agitateurs } \begin{array}{c} \\
\text { REZ-DE-CHAUSSÉE }\end{array} \\
\text { R.M. }\end{array}$ & 7 & 5 & 35 \\
\hline Ventilateur, laveuse et mécanisme... & 4 & 1,5 & 6 \\
\hline Pompe $\ldots \ldots \ldots \ldots \ldots \ldots \ldots$ & 3 & 1,5 & 4,5 \\
\hline Pompes à lait $\ldots \ldots \ldots \ldots \ldots \ldots$ & 4 & 3 & 12 \\
\hline $\begin{array}{c}\text { Pompe à lait acide } \ldots \ldots \ldots \ldots \ldots \ldots \\
\text { Sous-soL }\end{array}$ & 2 & 0,5 & 1 \\
\hline Divers $\quad \ldots \ldots \ldots \ldots \ldots \ldots \ldots$ & 4 & 1 & 4 \\
\hline Ecrémeuse, fromagerie . . . . . . . & 3 & 1 & 3 \\
\hline Pompe à sérum $\ldots \ldots \ldots \ldots \ldots$ & 1 & 1 & 1 \\
\hline
\end{tabular}




\begin{tabular}{|c|c|c|c|}
\hline Local et moteurs & Force & Durée & CV/Jour \\
\hline \multicolumn{4}{|l|}{ Pasteurisation } \\
\hline Pompe à eau chaude $\ldots \ldots \ldots \ldots \ldots$ & 2,5 & 3 & 7,5 \\
\hline Pompe à eau chaude nouvelle...... & 2,5 & 3 & 7,5 \\
\hline Pompe à lait $\ldots \ldots \ldots \ldots \ldots \ldots \ldots$ & 2 & 3 & 6 \\
\hline Pompe à lait nouvelle $\ldots \ldots \ldots \ldots \ldots$ & 2 & 3 & 6 \\
\hline Ecrémeuse $\quad \ldots \ldots \ldots \ldots \ldots \ldots \ldots$ & 5 & 3 & 15 \\
\hline $\begin{array}{c}\text { Eerémeuse nouvelle } \ldots \ldots \ldots \ldots \ldots \ldots \\
\text { Divers }\end{array}$ & \multicolumn{2}{|c|}{ Divers } & 15 \\
\hline Ventilation générale $\ldots \ldots \ldots \ldots \ldots$ & 5 & 4 & 20 \\
\hline Atelier $\ldots \ldots \ldots \ldots \ldots \ldots \ldots \ldots \ldots \ldots \ldots \ldots \ldots \ldots$ & 5 & 2 & 10 \\
\hline $\begin{array}{c}\text { Monte-charges } \ldots \ldots \ldots \ldots \ldots \ldots \ldots \\
\text { LAVAGE ET EMBouteIILAGE }\end{array}$ & 5 & 1 & 5 \\
\hline \multirow[t]{2}{*}{ Chaîne complète $\ldots \ldots \ldots \ldots \ldots \ldots$} & 15 & 5 & 75 \\
\hline & \multicolumn{3}{|c|}{ Total........ 720} \\
\hline $\begin{array}{ll}\text { Soit : } & 720 \mathrm{CVh} \text { par jour } \\
-\quad & 365 \times 720=262.800 \mathrm{CVh} \\
-\quad & 262.800 \times 0,736=193.420 \\
-\quad & 200.000 \mathrm{kWh} \text { (avec lumière) }\end{array}$ & an & & Avril 1952. \\
\hline
\end{tabular}

ANNEXe No 2

ÉTUde ÉCONOMIQUE CONGERNANT LE PROJET

(basée sur les prix pratiqués en mars 1952)

\section{I. - DONNÉES}

$1^{\circ}$ Quantité annuelle de lait à traiter dans l'usine envisagée

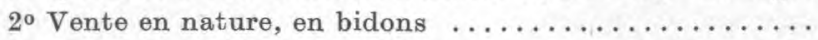
$3^{\circ}$ Vente en nature, en bouteilles..$\ldots \ldots \ldots \ldots \ldots \ldots$ $4^{0}$ Autres ventes . . . . . . . . . . . . . . . . . .

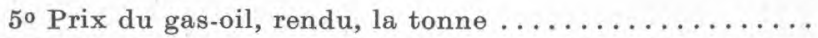

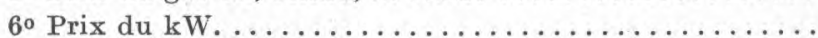

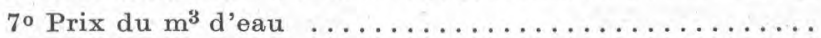

80 Prix d'achat des terrains et constructions existantes . .

90 Prix des travaux de bâtiments provisoires exécutés ...

$10^{\circ}$ Année de ces constructions . . . . . . . . . . . . .

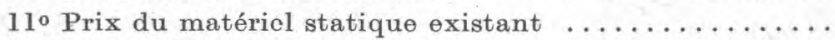

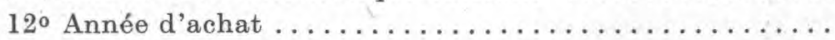

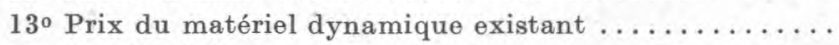

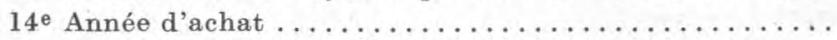

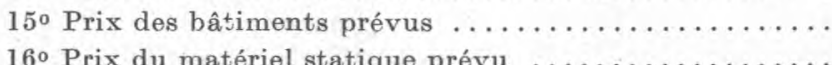

11.000 .0001$. 2.700.000 1. 5.300 .0001 . 3.000 .0001 . 12.5401.

$4.000 .000 "$

10.000 .000 "

1950

8.500 .000 "

1950

11.000 .000 "

1950

79.000 .000

26.500 .000 n 
170 Prix du matériel dynamique prévu

$33.500 .000 »$

$18^{\circ}$ Frais deramassage par litre d'après l'expérience ac quise y compris les amortissements et entretien du matériel roulant, du bidonnage et les frais de personnel .

$19^{\circ}$ Consommation de gas-oil :

- Pasteurisation (maximum 3 heures) $\ldots \ldots \ldots \ldots$

- Lavage bouteilles (moyenne 5 heures) ...........

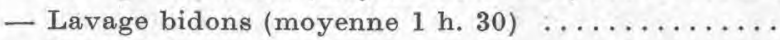

- Dirers

Total

Vapeur

$1.200 \mathrm{~kg}$.

$2.000 \mathrm{~kg}$.

$275 \mathrm{~kg}$.

$800 \mathrm{~kg}$.

$4.275 \mathrm{~kg}$.

de vapeur

Soit environ

$4.500 \mathrm{~kg}$.

- Soit environ :

$300 \mathrm{~kg}$. de gas-oil par jour,

110 tonnes de gas-oil par an.

$20^{\circ}$ Consommation électrique :

(voir tableau ei-joint des moteurs avec heures d'utilisation)

Total, par an

$200.000 \mathrm{kWh}$.

21e Eau (ville). - Etude faite avec récupérations (ruisselle. ment pour compresseurs et citernes), soit $120 \mathrm{~m}^{3}$ par jour restant, soit, annuels .................

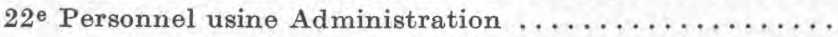

23e Personnel usine. - Main-d'œuvre intérieure 30 per-

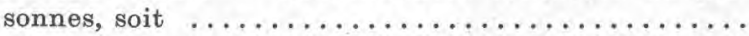

$24^{\circ}$ Coefficient d'amortissement réel, avec taux d'intérêt de $5 \%$ :

- Terrains et immeubles achetés 30 ans ..........

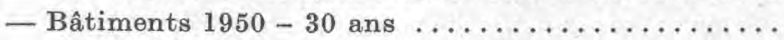

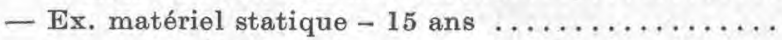

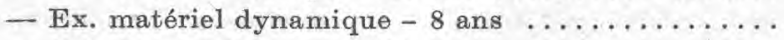

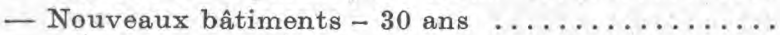

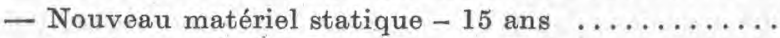

- Nouveau matériel dynamique -8 ans ...........

$-43.000 \mathrm{~m}^{3}$

4.000 .000

$15.000 .000 "$

0.015051

0.015051

0.046342

0.104722

0.015051

0.046342

0.104722

II. - CALCUL DES FRAIS ANNUELS BASÉ SUR LES PRIX DE MARS 1952

a) Amortissements.

1) Terrain :

2) Bâtiments 1950 :

$4.000 .000 \times 0.015051=$

60.204 "

3) Matériel statique installé en 1950 :

$8.500 .000 \times 0.046342=$ 150.510 ”

4) Matériel dynamique installé en 1950 :

393.907 "

5) Nouveaux bâtiments : $\begin{array}{r}11.000 .000 \times 0.104722= \\ 79.000 .000 \times 0.015051=\end{array}$ 
ESSAIS SUR EGHANTILLONS EXÉGUTÉS SUR PLACE

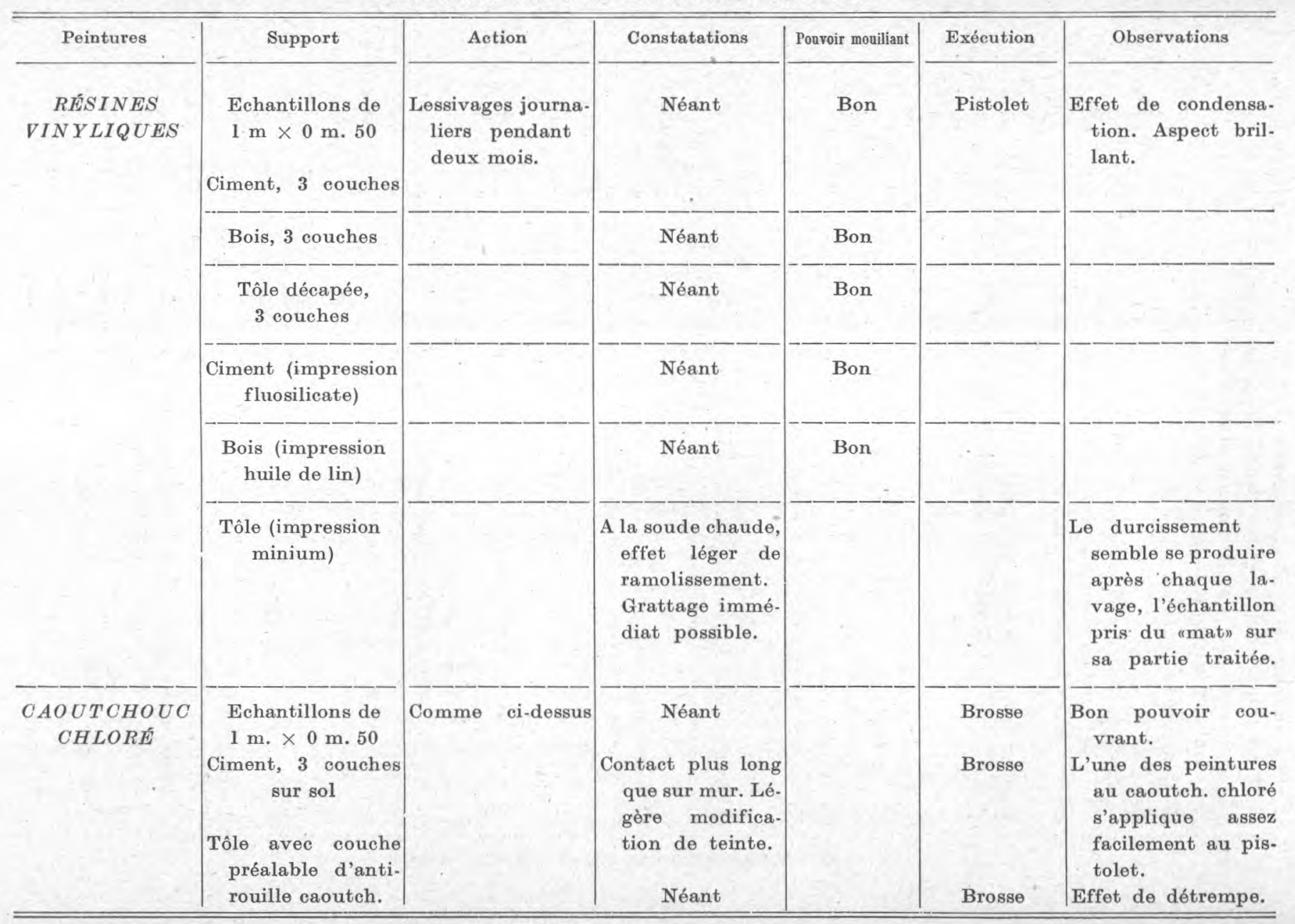


ESSAIS SUR ÉGHANTILLONS PRÉPARÉS A L'AVANGe

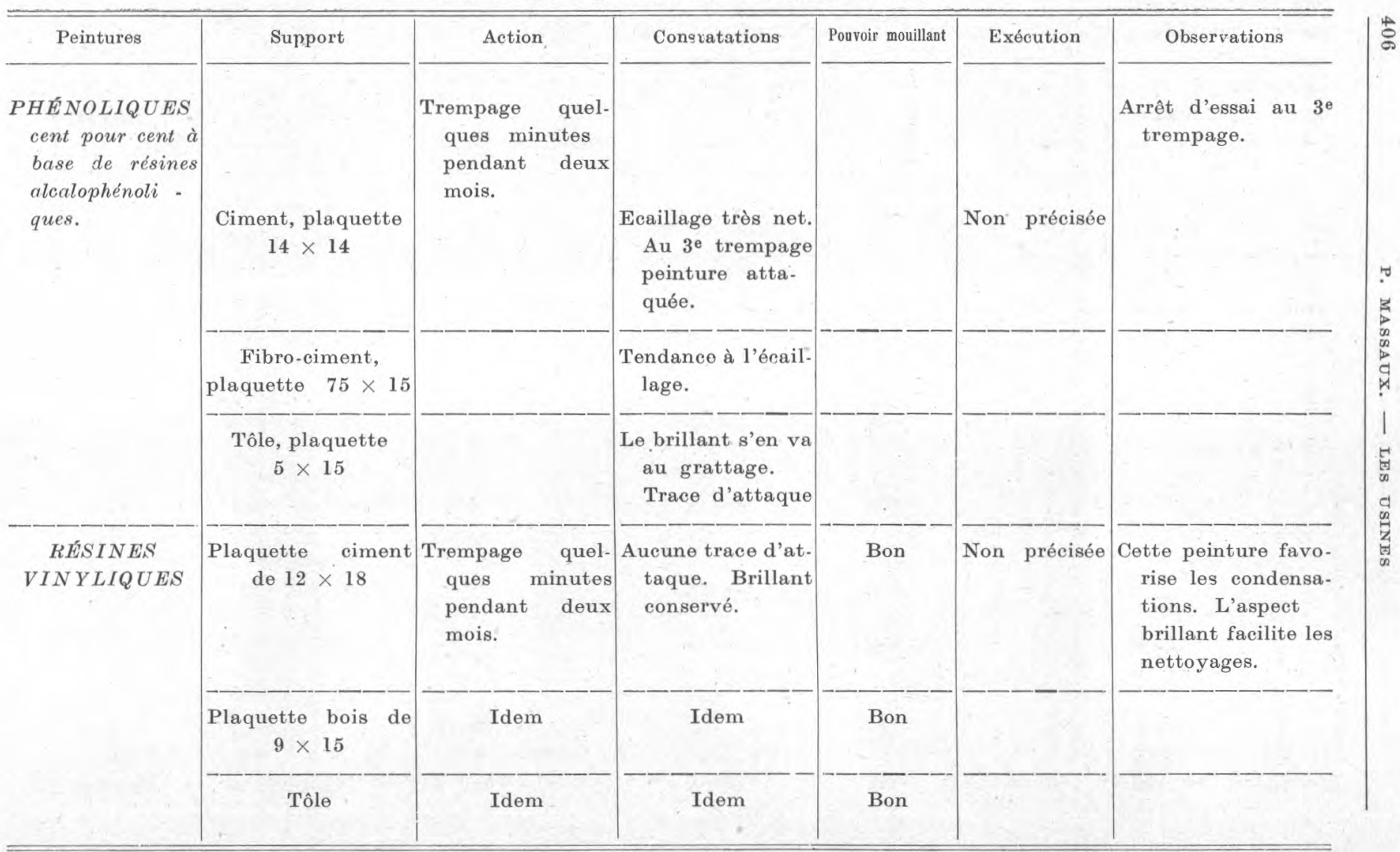


6) Matériel statique nouveau :

$$
26.500 .000 \times 0.046342=
$$

1.228 .063 ”

7) Matériel dynamique nouveau :

$$
33.500 .000 \times 0.104722=
$$

7.997 .913 》

Soit $\ldots \ldots \ldots \ldots \ldots \ldots \ldots \ldots$ 0,73 par litre

b) Exploitation

- Frais de ramassage :

$11.000 .0001 . \times 4.10=$

45.000 .000 ग

- Frais divers d'entretien (huile, produits de lavage, etc

- Consommation pour vapeur :

Gas-oil : 110 T. à $12.540=$

1.379 .400 »

- Consommation électricité : $\quad 200.000 \mathrm{kWh}$ à $7,53=$

- Consommation eau : $\quad 43.000 \mathrm{~m}^{3}$ à $17,00=$

- Personnel Administration ...............

$1.506 .000 \gg$ $744.600 \gg$

- Personnel intérieur usine ...............

- Frais de livraison, y compris remplacement de bouteilles, bidons, amortissements, etc. :

$$
\begin{aligned}
& \text { 11.000.000 1. à } 2,50= \\
& \text { Total } \ldots \ldots \ldots \ldots \ldots \ldots \ldots \ldots \ldots \\
& \text { Soit } \ldots \ldots \ldots \ldots \ldots \ldots
\end{aligned}
$$

\section{CONCLUSIONS}$$
4.000 .000 \gg
$$$$
15.000 .000 \gg
$$

- Les frais réels d'amortissement des installations sont

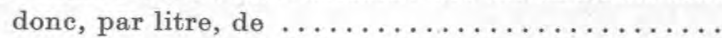

- Les frais réels d'exploitation sont de ..........

\section{ANNexe No 3}

\section{RAPPORT SUR ESSAIS DE PEINTURES POUR INDUSTRIES ALIMENTAIRES}

Le but de ces essais est une étude systématique de peintures pour l'industrie laitière et plus généralement pour les industries agricoles et alimentaires (frigorifiques, abattoirs, confitureries, sucreries, distilleries, etc.).

(Voir tableau ci-contre)

Les essais ont été effectués dans une laiterie, les échantillons étant soit de petites dimensions et préparés à l'avance par les fournisseurs, soit de grandes dimensions et exécutés sur place.

Les supports sont les suivants :

- Bois ;

- Tôle d'acier ordinaire ;

- Ciment.

Les produits chimiques d'attaque sont la soude diluée passée en solution utilisée normalement pour les lavages de bidons et bouteilles, et l'acide nitrique dilué du type solution de nettoyage des pasteurisateurs en eircuit fermé. 
Le traitement à la soude a été effectué quotidiennement à la brosse et pendant quelques minutes à chaque action. Cependant, les échantillons préparés en usine par les fournisseurs ont été trempés dans les bains de soude et non brossés.

Le traitement à l'acide azotique a eu lieu par aspersion.

Sur les échantillons de grandes dimensions, on n'a procédé au lavage que sur une moitié de chaque surface considérée, eeci permettant la comparaison des parties traitées et non traitées.

Nos constatations nous conduisent aux avis ci-dessous qui n'engagent évidemment que-les catégories de peintures étudiées dans le cadre des marques choisies.

Les peintures aux résines vinyliques et les peintures au caoutchouc chloré donnent satisfaction, mais il semble que les dernières soient d'une application moins facile. Les peintures phénoliques résistent moins aux acides et bases diluśes que les précédentes, leur applieation est très aisée. Dans la mesure où la détrempe n'existe pas pour les peintures phénoliques, on peut les utiliser pour des revêtements de murs, mais l'utilisation dans deś baes de stockage de produits acides ou d'alcali ne nous parait pas à conseiller.

Dans tous les cas, une couche de protection antirouille est nécessaire sur tôle.

Sur bois, nos essais sont assez réduits, les résultats pouvant varier avec l'essence choisie. On attachera pour ce matériau une grande importance à l'élasticité de la peinture (effet de l'humidité sur le bois et craquelures).

Sur eiment, une couch'́ de protection au fluosilicate est à conseiller.

Si nous pouvons nous permettre un classement à la suite de ces essais, nous proposons :

- 10 Résines vinyliques;

- 20 Caoutehoue chloré;

- $3^{\circ}$ Phénolique cent pour cent.

\title{
UTILISATION DU LAIT DE BREBIS \\ EN ISRAEL (1)
}

\author{
par \\ le $\mathrm{D}^{r} \mathrm{KERN}$
}

Comme on l'a vu dans un premier article (2), on a réussi par un élevage méthodique à obtenir, en partant de la brebis primitive à grosse queue, une brebis laitière produisant presque autant de lait qu'une vache primitive.

Quand il est question des brebis laitières et de leurs produits, les lecteurs européens se souviennent tout d'abord du Roquefort français, du "Liptauer " des contrées carpathiques, du Caccio-

(1) Traduction C. Wolf, à qui Le Lait exprime à nouveau sa profonde gratitude.

(2) Le Lait, 1954, 335-336. 